\title{
Solutions of fractional logistic equations by Euler's numbers
}

\author{
Mirko D’Ovidio $^{\mathrm{a}, *}$, Paola Loreti $^{\mathrm{b}}$ \\ a Department of Basic and Applied Sciences for Engineering, Sapienza University of Rome, via A. Scarpa 10, Rome, Italy \\ ${ }^{b}$ Department of Basic and Applied Sciences for Engineering, Sapienza University of Rome, via A. Scarpa 16, Rome, Italy
}

\section{A R T I C L E I N F O}

\section{Article history:}

Received 21 December 2017

Received in revised form 14 March 2018

Available online 12 May 2018

\section{MSC:}

11B68

78A70

$26 \mathrm{A3} 3$

Keywords:

Euler's numbers

Biological application

Fractional logistic equation

\begin{abstract}
A B S T R A C T
In this paper, we solve in the convergence set, the fractional logistic equation making use of Euler's numbers. To our knowledge, the answer is still an open question. The key point is that the coefficients can be connected with Euler's numbers, and then they can be explicitly given. The constrained of our approach is that the formula is not valid outside the convergence set. The idea of the proof consists to explore some analogies with logistic function and Euler's numbers, and then to generalize them in the fractional case.
\end{abstract}

(C) 2018 Elsevier B.V. All rights reserved.

\section{Introduction}

\subsection{The logistic function}

A logistic function is

$$
u(t)=\frac{u_{0}}{u_{0}+\left(1-u_{0}\right) e^{-t / M}}
$$

with $M$ positive and $u_{0}$ positive and less than 1 . The function was introduced by Pierre Francois Verhulst [1] to model the population growth. At the beginning of the process the growth of the population is fast; then, as saturation process begins, the growth slows, and then growth is close to be flat.

The logistic function is solution of the logistic differential equation

$$
u^{\prime}(t)=\frac{1}{M}\left(u(t)-u^{2}(t)\right)
$$

with initial condition

$$
u(0)=u_{0} .
$$

The key assumptions in the logistic model are:

- The population is composed by individual not distinguishable;

\footnotetext{
* Corresponding author.

E-mail addresses: mirko.dovidio@uniroma1.it (M. D’Ovidio), paola.loreti@uniroma1.it (P. Loreti).
} 
- The population is isolated;

- Self-limiting growth, that is an intrinsic mechanism of saturation holds when the density of population reaches a certain level.

These basic assumptions may be checked in laboratory for biological diffusion. At least for bounded time they agree to the experience, hence they may be adopted to describe phenomena as biological models of tumour growth [2-5]. As well many processes may be modelled by the logistic differential equation, or generalization of it, and the applications are wide and in different field of applications [6].

Sharing the applications with the logistic case, the fractional equation is a model for the growth of a given population, describing the population behaviour and showing an increase, a saturation and a flat asymptotic behaviour. The global shape is also respected by the fractional logistic case by numerical evidence; however, some peculiar differences show that the fractional model is a good candidate to model a memory effect on the population (see (1.7)) and that the fractional order may be modified along the process in order to constrain the growth (see for example [7] for the estimation of fractional order by observations).

The problem to give a solution of the fractional logistic equation was unsolved and several attempts have been done (see for instance [8-11]). A close answer from an empirical point of view is contained in [12]. The solution we propose here has an exact representation given by a closed formula for the coefficients in the convergence set. The convergence analysis is stated in Theorem 3.1

A model considering a modified logistic equation has been also treated in [13].

To our knowledge, from a mathematical point of view, the fractional logistic function is undefined. This paper answers to the question for limited time. The observation is that for any positive $t$ less than $\pi$, denoted by $u$ the logistic function (with all the parameters simplified) the equality holds true

$$
u(t)=\frac{1}{2} \sum_{k \geq 0} \frac{E_{k}}{k !} t^{k},
$$

with $E_{k}$ the Euler's numbers. In the next subsection we explore this point.

\subsection{The logistic fractional function}

In this subsection we introduce the logistic fractional function. As usual by $\Gamma$ we denote

$$
\Gamma(x)=\int_{0}^{+\infty} t^{x-1} e^{-t} d t
$$

for any real positive $x$.

Let $R>0$ fixed. In $(0, R)$, we introduce the function

$$
w(t)=\sum_{k \geq 0} \frac{E_{k}^{\beta}}{M^{\beta k}} \frac{t^{\beta k}}{\Gamma(\beta k+1)}, \quad M \geq 1, \quad \beta \in(0,1), \quad t<R
$$

where the Euler's $\beta$-numbers $\left\{E_{k}^{\beta}\right\}_{k \in \mathbb{N}_{0}}$ are defined here by considering the relation

$$
\begin{aligned}
E_{k+1}^{\beta} & =-\sum_{\substack{i, j \\
i+j=k}} \frac{\Gamma(\beta k+1)}{\Gamma(\beta i+1) \Gamma(\beta j+1)} E_{i}^{\beta} E_{j}^{\beta} \\
& =-\frac{1}{\beta k+1} \sum_{\substack{i, j \\
i+j=k}} \frac{E_{i}^{\beta} E_{j}^{\beta}}{B(\beta i+1, \beta j+1)}, \quad k \in 2 \mathbb{N}
\end{aligned}
$$

together with the logistic constrains

$$
\begin{aligned}
& E_{1}^{\beta}=E_{0}^{\beta}-\left(E_{0}^{\beta}\right)^{2}, \\
& E_{0}^{\beta}=1 / 2 .
\end{aligned}
$$

The result we obtain is that, in the convergence set, the function $w$ is solution (in the Caputo sense) of the fractional logistic equation. We recall that the Caputo fractional derivative is given by

$$
{ }^{*} D_{t}^{\beta} w(t):=\frac{1}{\Gamma(1-\beta)} \int_{0}^{t} \frac{w^{\prime}(s)}{(t-s)^{\beta}} d s, \quad t>0, \quad \beta \in(0,1)
$$

with $w^{\prime}(s)=d w / d s$. Thus, the fractional logistic equation is

$$
{ }^{*} D_{t}^{\beta} w=\frac{1}{M^{\beta}} w(1-w)
$$


for $M=1$. The proof of this result leads to introduce a new class of numbers that corresponds to Euler's numbers for special value of the fractional order. This new class that we called generalized Euler's numbers has an interest by itself. As we will see in the paper they share with the Euler's numbers properties helpful to find solutions. The method we propose here is suggested by the logistic case and goes back to the exact solution to the logistic equation in $(0, \pi)$. However, in the fractional logistic case other considerations are no more valid to solve the problem in the whole positive line.

The fractional equation (1.6) has the following interesting integro-differential counterpart

$$
\frac{d w}{d t}=\frac{1}{M^{\beta}} \frac{1}{\Gamma(\beta)} \int_{0}^{t}\left(w(s)-w^{2}(s)\right)(t-s)^{\beta-1} d s
$$

that we also consider further on.

\section{Logistic equation and fractional derivatives}

\subsection{The Euler's numbers}

We first recall the Euler's polynomials

$$
E_{k}(x)=\frac{1}{k+1} \sum_{s=0}^{k}\left(\begin{array}{c}
k+1 \\
s
\end{array}\right) 2^{s} B_{s}(x / 2)
$$

where the Bernoulli polynomials can be written as

$$
B_{s}(x)=\sum_{j=0}^{s}\left(\begin{array}{l}
s \\
j
\end{array}\right) b_{s-j} x^{j}
$$

in terms of the Bernoulli numbers $b_{j}=B_{j}(0)$ (usually denoted by $B_{j}^{-}$). In particular,

$$
b_{0}=1, \quad b_{1}=-\frac{1}{2}, \quad b_{2}=\frac{1}{6}, \quad b_{3}=0, \quad b_{4}=-\frac{1}{30}, \quad b_{5}=0, \quad b_{6}=\frac{1}{42}, \quad b_{7}=0, \quad b_{8}=-\frac{1}{30}, \ldots
$$

We recall the useful formula

$$
E_{k}(x)=\sum_{s=0}^{k}\left(\begin{array}{l}
k \\
s
\end{array}\right) \frac{E_{k}}{2^{k}}\left(x-\frac{1}{2}\right)^{k} .
$$

We also recall some explicit case of the Euler's polynomial

$$
E_{0}(x)=1, \quad E_{1}(x)=x-\frac{1}{2}, \quad E_{2}(x)=x^{2}-x, \quad E_{3}(x)=x^{2}-\frac{3}{2} x^{2}+\frac{1}{4}, \quad E_{4}(x)=x^{4}-2 x^{3}+x, \ldots
$$

and the Euler's numbers are usually referred to as $2^{k} E_{k}\left(\frac{1}{2}\right)$. Here, we consider the Euler's polynomials at $x=1$ and, in particular we have that the Euler's numbers $E_{k}=E_{k}(1)$ are given by

$$
E_{0}=1, \quad E_{1}=\frac{1}{2}, \quad E_{2}=0, \quad E_{3}=-\frac{1}{4}, \quad E_{4}=0, \ldots
$$

We refer to the book [14, formulas from no. 9.6] for details on this topic.

\subsection{The logistic equation}

Let us consider the generating function ([14, formula no. 9.65])

$$
\frac{e^{x t}}{1+e^{t}}=\frac{1}{2} \sum_{k \geq 0} E_{k}(x) \frac{t^{k}}{k !}, \quad|t|<\pi
$$

of the Euler's polynomials $E_{k}(x)$ introduced above. For $k$ odd, we have that (see [15, Corollary 3])

$$
(-1)^{\frac{k+1}{2}} \frac{\pi^{k+1}}{4 \Gamma(k+1)} E_{k}(x) \rightarrow \cos (\pi x) \quad \text { as } k \rightarrow \infty .
$$

We immediately recover the uniform convergence for the series (2.1) on compact subsets of $\mathbb{C}$.

For the sake of simplicity we now assume that $M=1$ in (1.1). Throughout, we consider the function (1.1) written as in (1.2), that is formula (2.1) with $x=1$. This is a special case obtained form (3.1) with $\beta=1$. Indeed, we show that, for $\beta=1$,

$$
E_{k}^{1}=\frac{E_{k}}{2}=\frac{E_{k}(1)}{2}
$$

where $E_{k}=E_{k}(1)$ are the Euler's polynomials evaluated at $x=1$. 
Remark 2.1. Observe that,

$$
U(t)=\sum_{k \geq 0}\left(\frac{a}{g(t)}\right)^{k}=\frac{g(t)}{a+g(t)}=\left(1+e^{-\int_{0}^{t} v(s) d s}\right)^{-1}, \quad g(t)<a
$$

where $v(s)=(\ln g(s))^{\prime} \mathbf{1}_{(g(s)>a)}$ solves the logistic equation

$$
U^{\prime}=(\ln g)^{\prime}\left(U-U^{2}\right), \quad g(t)<a .
$$

For the sake of simplicity we equals to one the growth rate and the carrying capacity considered in the literature. Thus, $(1-u)$ is the biological potential describing the density dependence, if negative, the population decreases back to the carrying capacity. The logistic model may be generalized as follow

$$
K^{\Phi} * u^{\prime}=f(u)
$$

where

$$
\left(K^{\Phi} * u^{\prime}\right)(t):=\int_{0}^{t} K^{\Phi}(t-s) u^{\prime}(s) d s .
$$

and

$$
f(u)=u(1-u)
$$

Definition 1. A function $\phi:(0, \infty) \mapsto \mathbb{R}$ is a Bernstein function if $\phi$ is of class $C^{\infty}, \phi(\lambda) \geq 0$ for all $\lambda>0$ and

$$
-(-1)^{n} \phi^{(n)}(\lambda) \geq 0 \text { for all } n \in \mathbb{N} \text { and } \lambda>0 .
$$

We introduce the Bernstein function

$$
\Phi(\lambda)=\int_{0}^{\infty}\left(1-e^{-\lambda z}\right) \Pi(d z)
$$

with

$$
\frac{\Phi(\lambda)}{\lambda}=\int_{0}^{\infty} e^{-\lambda z} \Pi((z, \infty)) d z
$$

where $\Pi$ on $(0, \infty)$ with $\int(1 \wedge z) \Pi(d z)<\infty$ is the associated Lévy measure (and $\Pi((z, \infty))$ is the tail of the Lévy measure $\Pi)$. If $\Phi(\lambda)=\lambda^{\beta}$ for instance, we have that

$$
\Pi(d z)=\frac{\beta}{\Gamma(1-\beta)} z^{-\beta-1} d z \text { and } \Pi((z, \infty))=\frac{z^{-\beta}}{\Gamma(1-\beta)} .
$$

Let $M>0$ and $w \geq 0$. Let $\mathcal{M}_{\omega}$ be the set of (piecewise) continuous function on $[0, \infty)$ of exponential order $\omega$ such that $|u(t)| \leq M e^{\omega t}$. Denote by $\widetilde{u}$ the Laplace transform of $u$. Then, we define the operator $\mathfrak{D}_{t}^{\Phi}: \mathcal{M}_{\omega} \mapsto \mathcal{M}_{\omega}$ such that

$$
\int_{0}^{\infty} e^{-\lambda t} \mathfrak{D}_{t}^{\Phi} u(t) d t=\Phi(\lambda) \widetilde{u}(\lambda)-\frac{\Phi(\lambda)}{\lambda} u(0), \quad \lambda>\omega
$$

where $\Phi$ is given in (2.4). Since $u$ is exponentially bounded, the integral $\tilde{u}$ is absolutely convergent for $\lambda>\omega$. By Lerch's theorem the inverse Laplace transforms $u$ and $\mathfrak{D}_{t}^{\Phi} u$ are uniquely defined. Since

$$
\Phi(\lambda) \widetilde{u}(\lambda)-\frac{\Phi(\lambda)}{\lambda} u(0)=(\lambda \widetilde{u}(\lambda)-u(0)) \frac{\Phi(\lambda)}{\lambda}
$$

the operator $\mathfrak{D}_{t}^{\Phi}$ can be written as a convolution involving the ordinary derivative and the inverse transform of $(2.5)$ iff $u \in \mathcal{M}_{\omega} \cap C\left([0, \infty), \mathbb{R}_{+}\right)$and $u^{\prime} \in \mathcal{M}_{\omega}$. Throughout we consider

$$
\mathfrak{D}_{t}^{\Phi} u=f(u) \text {. }
$$

For different definitions and representations of the operator $\mathfrak{D}_{t}^{\Phi}$ the interested reader can also see the recent works [16-18] and $[19,20]$ for fractional calculus and derivatives.

Remark 2.2. We observe that for $\Phi(\lambda)=\lambda$ (that is we deal with the ordinary derivative) we have that Eq. (2.7) becomes the logistic equation.

Remark 2.3. If $\Phi(\lambda)=\lambda^{\beta}$, the operator $\mathfrak{D}_{t}^{\Phi}$ becomes the Caputo fractional derivative

$$
\mathfrak{D}_{t}^{\Phi} u(t)={ }^{*} D_{t}^{\beta} u(t):=\frac{1}{\Gamma(1-\beta)} \int_{0}^{t} \frac{u^{\prime}(s)}{(t-s)^{\beta}} d s, \quad t>0, \quad \beta \in(0,1)
$$


with $u^{\prime}(s)=d u / d s$. Thus, Eq. (2.7) becomes the equation

$$
{ }^{*} D_{t}^{\beta} u=f(u)
$$

which is well-known in the literature as the fractional logistic equation.

Remark 2.4. A further example is given by the symbol $\Phi(\lambda)=\lambda^{2 \beta}+\lambda^{\beta}$ for $\beta \in(0,1 / 2)$, that is, $\mathfrak{D}_{t}^{\Phi}$ becomes the telegraph fractional operator. Since we have infinite Bernstein function, then we can define a number of fractional operators together with the correspond fractional equations (2.7).

We denote by $D_{t}^{\beta}$ the Riemann-Liouville derivative

$$
D_{t}^{\beta} u(t):=\frac{1}{\Gamma(1-\beta)} \frac{d}{d t} \int_{0}^{t} \frac{u(s)}{(t-s)^{\beta}} d s, \quad t>0, \quad \beta \in(0,1)
$$

with Laplace transform

$$
\int_{0}^{\infty} e^{-\lambda t} D_{t}^{\beta} u(t) d t=\lambda^{\beta} \widetilde{u}(\lambda)
$$

We recall that

$$
\int_{0}^{\infty} e^{-\lambda t *} D_{t}^{\beta} u(t) d t=\lambda^{\beta} \widetilde{u}(\lambda)-\lambda^{\beta-1} u(0) .
$$

We also recall the Stirling's approximation for the Gamma function

$$
\Gamma(x):=\int_{0}^{\infty} e^{-z} z^{x-1} d z \sim \sqrt{2 \pi x}\left(\frac{x}{e}\right)^{x}
$$

and the Beta function

$$
B(x, y):=\int_{0}^{1} z^{x-1}(1-z)^{y-1} d z \sim \sqrt{2 \pi} \frac{x^{x-1 / 2} y^{y-1 / 2}}{(x+y)^{x+y-1 / 2}}
$$

for large values of $x>0$ and $y>0$. Moreover, the following bounds hold true [21]

$$
B(x, y) \leq \frac{1}{x y}, \quad x, y>1
$$

and [22]

$$
2^{x-1} \leq \Gamma(x+1) \leq 1, \quad 0 \leq x \leq 1 .
$$

We also consider the following bounds for the Gamma function [23]

$$
\frac{x^{x-\gamma}}{e^{x-1}}<\Gamma(x)<\frac{x^{x-1 / 2}}{e^{x-1}}, \quad x>1
$$

where

$$
\gamma \approx 0.577215 \text {. }
$$

is the Euler-Mascheroni constant. Note that, from (2.9),

$$
\left.\Gamma(1-\beta)\right|^{*} D_{t}^{\beta} u\left|\leq \int_{0}^{t}\right| u^{\prime}(s) \mid(t-s)^{-\beta} d s \leq B(1+\theta, 1-\beta) \quad \text { if } \quad\left|u^{\prime}(t)\right| \leq t^{\theta}, \theta>-1
$$

and, from (2.11) and the fact that $(1-\beta) \Gamma(1-\beta)=\Gamma(2-\beta)$ we have that

$$
\left.\right|^{*} D_{t}^{\beta} u \mid \leq(1-\beta) B(1+\theta, 1-\beta) \leq B(1+\theta, 1-\beta) .
$$

From the Laplace transform (2.6), by Young's inequality, we obtain that

$$
\int_{0}^{\infty}\left|\mathfrak{D}_{t}^{\Phi} u\right|^{p} d t \leq\left(\int_{0}^{\infty}\left|u^{\prime}\right|^{p} d t\right)\left(\lim _{\lambda \downarrow 0} \frac{\Phi(\lambda)}{\lambda}\right)^{p}, \quad p \in[1, \infty)
$$

where $\lim _{\lambda \downarrow 0} \Phi(\lambda) / \lambda$ is finite only in some cases. 


\section{Fractional logistic equation: Main result and proof}

\subsection{The fractional equation}

Let $R>0$. We consider the function

$$
w(t)=\sum_{k \geq 0} \frac{E_{k}^{\beta}}{M^{\beta k}} \frac{t^{\beta k}}{\Gamma(\beta k+1)}, \quad M \geq 1, \quad \beta \in(0,1), \quad t<R
$$

where

$$
\begin{aligned}
E_{k+1}^{\beta} & =-\sum_{\substack{i, j \\
i+j=k}} \frac{\Gamma(\beta k+1)}{\Gamma(\beta i+1) \Gamma(\beta j+1)} E_{i}^{\beta} E_{j}^{\beta} \\
& =-\frac{1}{\beta k+1} \sum_{\substack{i, j \\
i+j=k}} \frac{E_{i}^{\beta} E_{j}^{\beta}}{B(\beta i+1, \beta j+1)}, \quad k \in 2 \mathbb{N}
\end{aligned}
$$

with the logistic constrains

$$
\begin{aligned}
& E_{1}^{\beta}=E_{0}^{\beta}-\left(E_{0}^{\beta}\right)^{2}, \\
& E_{0}^{\beta}=1 / 2 .
\end{aligned}
$$

We refer to the sequence of Euler's fractional numbers $\left\{E_{n}^{\beta}\right\}_{n \in \mathbb{N}_{0}}$ as the Euler's $\beta$-numbers (we introduce the Euler's $\Phi$ number further on).

We are not able to prove absolute or uniform convergence of $(3.1)$ in $(0, R)$. Our approach is therefore focused on the Weierstrass M-test in order to obtain different intervals of convergence depending on the fractional order $\beta$ for different values of $\beta$. Thus, we obtain that (3.1) is convergent in some subsets of $(0, R)$, that is $w(t)$ is absolutely convergent $\forall t \in\left(0, r_{\beta}\right)$. In particular, we have uniform convergence in $\left(0, r_{\beta}\right)$. Each interval of convergence turns out to be given in terms of $\beta \in(0,1]$ and it depends continuously on $\beta$. As $\beta$ increases to one we recover the solution to the logistic equation and the convergence in $(0, \pi)$ of $u^{\prime}$ and $u$ in (1.2) has been extensively investigated in the literature. Concerning the differentiation of $w$ for $\beta \in(0,1)$ we easily have convergence on $\left(0, r_{\beta}\right)$ from $(1.6)$ and the uniform convergence of the product of uniformly convergent series.

Theorem 3.1. Let $\beta \in(0,1), M>1,0<r_{\beta}<R$. Let $\gamma$ be the Euler-Mascheroni constant. The series (3.1) uniformly converges in each compact subsets of $\left(0, r_{\beta}\right)$. In particular,

(i) we have that

$$
r_{\beta}=M\left(\frac{\Gamma(\beta+1) \Gamma(3 \beta+1)}{\Gamma(2 \beta+1)}\right)^{\frac{1}{2 \beta}}
$$

and $r_{\beta} \downarrow M$ as $\beta \downarrow 0, r_{\beta} \uparrow M c_{1}$ as $\beta \uparrow 1$ where $c_{1} \approx 1.732051$.

(ii) Moreover,

$$
r_{\beta} \geq \rho_{\beta}=M\left(\frac{2^{\beta}}{e^{\beta}}\left(\frac{3 \beta+1}{2 \beta+1}\right)^{2 \beta+1 / 2}(3 \beta+1)^{\beta+(1 / 2-\gamma)}\right)^{\frac{1}{2 \beta}}
$$

and $\rho_{\beta} \uparrow M c_{2}$ as $\beta \uparrow 1$ where $c_{2} \approx 1.647524$.

The series (3.1) solves the fractional logistic equation

$$
{ }^{*} D_{t}^{\beta} w(t)=\frac{1}{M^{\beta}} w(t)(1-w(t)), \quad w(0)=1 / 2 .
$$

Proof. We first show that (3.1) solves (3.4) pointwise and then we study the convergence of (3.1).

(1) The Riemann-Liouville derivative of $w$ gives the series

$$
D_{t}^{\beta} w(t)=\sum_{k \geq 0} \frac{E_{k}^{\beta}}{M^{\beta} k} \frac{t^{\beta k-\beta}}{\Gamma(\beta k+1-\beta)}=E_{0}^{\beta} \frac{t^{-\beta}}{\Gamma(1-\beta)}+\sum_{k \geq 1} \frac{E_{k}^{\beta}}{M^{\beta k}} \frac{t^{\beta k-\beta}}{\Gamma(\beta k+1-\beta)}
$$

that is, the Caputo derivative of $w$ is given by

$$
{ }^{*} D_{t}^{\beta} w(t)=D_{t}^{\beta}(w(t)-w(0))=\sum_{k \geq 0} \frac{E_{k+1}^{\beta}}{M^{\beta(k+1)}} \frac{t^{\beta k}}{\Gamma(\beta k+1)} .
$$


Let $L w=w-w^{2}$ be the logistic operator. From (3.1) we have that

$$
\begin{aligned}
L w(t)= & \sum_{k \geq 0} \frac{E_{k}^{\beta}}{M^{\beta k}} \frac{t^{\beta k}}{\Gamma(\beta k+1)}-\sum_{k \geq 0} \sum_{s \geq 0} \frac{E_{k}^{\beta}}{M^{\beta k}} \frac{t^{\beta k}}{\Gamma(\beta k+1)} \frac{E_{s}^{\beta}}{M^{\beta s}} \frac{t^{\beta s}}{\Gamma(\beta s+1)} \\
= & E_{0}^{\beta}-E_{0}^{\beta} E_{0}^{\beta} \\
& +\left(\frac{E_{1}^{\beta}}{\Gamma(\beta+1)}-2 \frac{E_{0}^{\beta} E_{1}^{\beta}}{\Gamma(\beta+1)}\right) \frac{t^{\beta}}{M^{\beta}} \\
& +\left(\frac{E_{2}^{\beta}}{\Gamma(2 \beta+1)}-2 \frac{E_{0}^{\beta} E_{2}^{\beta}}{\Gamma(2 \beta+1)}-\frac{E_{1}^{\beta} E_{1}^{\beta}}{\Gamma(\beta+1) \Gamma(\beta+1)}\right) \frac{t^{2 \beta}}{M^{2 \beta}} \\
& +\left(\frac{E_{3}^{\beta}}{\Gamma(3 \beta+1)}-2 \frac{E_{0}^{\beta} E_{3}^{\beta}}{\Gamma(3 \beta+1)}-2 \frac{E_{1}^{\beta} E_{2}^{\beta}}{\Gamma(\beta+1) \Gamma(2 \beta+1)}\right) \frac{t^{3 \beta}}{M^{3 \beta}} \\
& +\left(\frac{E_{4}^{\beta}}{\Gamma(4 \beta+1)}-2 \frac{E_{0}^{\beta} E_{4}^{\beta}}{\Gamma(4 \beta+1)}-2 \frac{E_{1}^{\beta} E_{3}^{\beta}}{\Gamma(\beta+1) \Gamma(3 \beta+1)}-\frac{E_{2}^{\beta} E_{2}^{\beta}}{\Gamma(2 \beta+1) \Gamma(2 \beta+1)}\right) \frac{t^{4 \beta}}{M^{4 \beta}} \\
& +\ldots
\end{aligned}
$$

Under the constrains (3.3) we obtain the coefficients $E_{k}^{\beta}$ for $k>1$. In particular, we have that

$$
\begin{aligned}
\frac{1}{M^{\beta}} \frac{E_{2}^{\beta}}{\Gamma(\beta+1)} & =\frac{E_{1}^{\beta}}{\Gamma(\beta+1)}-2 \frac{E_{0}^{\beta} E_{1}^{\beta}}{\Gamma(\beta+1)} \\
\frac{1}{M^{\beta}} \frac{E_{3}^{\beta}}{\Gamma(2 \beta+1)} & =-\frac{E_{1}^{\beta} E_{1}^{\beta}}{\Gamma(\beta+1) \Gamma(\beta+1)} \\
\frac{1}{M^{\beta}} \frac{E_{4}^{\beta}}{\Gamma(3 \beta+1)} & =\frac{E_{3}^{\beta}}{\Gamma(3 \beta+1)}-2 \frac{E_{0}^{\beta} E_{3}^{\beta}}{\Gamma(3 \beta+1)} \\
\frac{1}{M^{\beta}} \frac{E_{5}^{\beta}}{\Gamma(4 \beta+1)} & =-2 \frac{E_{1}^{\beta} E_{3}^{\beta}}{\Gamma(\beta+1) \Gamma(3 \beta+1)} \\
\frac{1}{M^{\beta}} \frac{E_{6}^{\beta}}{\Gamma(5 \beta+1)} & =\frac{E_{5}^{\beta}}{\Gamma(5 \beta+1)}-2 \frac{E_{0}^{\beta} E_{5}^{\beta}}{\Gamma(5 \beta+1)} \\
\frac{1}{M^{\beta}} \frac{E_{7}^{\beta}}{\Gamma(6 \beta+1)} & =-2 \frac{E_{1}^{\beta} E_{5}^{\beta}}{\Gamma(\beta+1) \Gamma(5 \beta+1)}-\frac{E_{3}^{\beta} E_{3}^{\beta}}{\Gamma(3 \beta+1) \Gamma(3 \beta+1)} \\
\frac{1}{M^{\beta}} \frac{E_{8}^{\beta}}{\Gamma(7 \beta+1)} & =\frac{E_{7}^{\beta}}{\Gamma(7 \beta+1)}-2 \frac{E_{0}^{\beta} E_{7}^{\beta}}{\Gamma(7 \beta+1)} \\
\frac{1}{M^{\beta}} \frac{E_{9}^{\beta}}{\Gamma(8 \beta+1)} & =-2 \frac{E_{1}^{\beta} E_{7}^{\beta}}{\Gamma(\beta+1) \Gamma(7 \beta+1)}-2 \frac{E_{3}^{\beta} E_{5}^{\beta}}{\Gamma(3 \beta+1) \Gamma(5 \beta+1)} \\
\frac{1}{M^{\beta}} \frac{E_{11}^{\beta}}{\Gamma(10 \beta+1)} & =-2 \frac{E_{1}^{\beta} E_{9}^{\beta}}{\Gamma(\beta+1) \Gamma(9 \beta+1)}-2 \frac{E_{3}^{\beta} E_{7}^{\beta}}{\Gamma(3 \beta+1) \Gamma(7 \beta+1)}-\frac{E_{5}^{\beta} E_{5}^{\beta}}{\Gamma(5 \beta+1) \Gamma(5 \beta+1)}
\end{aligned}
$$

that is

$$
\frac{1}{M^{\beta}} E_{n+1}^{\beta}=-\sum_{\substack{i, j \\ i+j=n}} \frac{\Gamma(n \beta+1)}{\Gamma(i \beta+1) \Gamma(j \beta+1)} E_{i}^{\beta} E_{j}^{\beta} .
$$

As a consequence of the logistic conditions (3.3) we have that $E_{2 k}^{\beta}=0$.

(2) Now we prove uniform convergence of (3.1). We first rewrite the coefficient $E_{k}^{\beta}$ as follows

$$
\begin{aligned}
& E_{1}^{\beta}=+\Gamma(\beta+1)\left(\frac{E_{1}^{\beta}}{\Gamma(\beta+1)}\right)=1 / 4 \\
& E_{3}^{\beta}=-\Gamma(2 \beta+1)\left(\frac{E_{1}^{\beta}}{\Gamma(\beta+1)}\right)^{2} \\
& E_{5}^{\beta}=+2 \Gamma(4 \beta+1) \frac{\Gamma(2 \beta+1)}{\Gamma(3 \beta+1)}\left(\frac{E_{1}^{\beta}}{\Gamma(\beta+1)}\right)^{3}
\end{aligned}
$$




$$
\begin{aligned}
E_{7}^{\beta}= & -2^{2} \Gamma(6 \beta+1)\left[\frac{\Gamma(4 \beta+1) \Gamma(2 \beta+1)}{\Gamma(5 \beta+1) \Gamma(3 \beta+1)}+\frac{1}{4}\left(\frac{\Gamma(2 \beta+1)}{\Gamma(3 \beta+1)}\right)^{2}\right]\left(\frac{E_{1}^{\beta}}{\Gamma(\beta+1)}\right)^{4} \\
E_{9}^{\beta}= & +2^{3} \Gamma(8 \beta+1)\left[\frac{\Gamma(6 \beta+1) \Gamma(4 \beta+1) \Gamma(2 \beta+1)}{\Gamma(7 \beta+1) \Gamma(5 \beta+1) \Gamma(3 \beta+1)}+\frac{1}{2} \frac{\Gamma(4 \beta+1)}{\Gamma(5 \beta+1)}\left(\frac{\Gamma(2 \beta+1)}{\Gamma(3 \beta+1)}\right)^{2}+\right. \\
& \left.+2^{3} \Gamma(8 \beta+1) \quad+\frac{1}{4} \frac{\Gamma(6 \beta+1)}{\Gamma(7 \beta+1)}\left(\frac{\Gamma(2 \beta+1)}{\Gamma(3 \beta+1)}\right)^{2}\right]\left(\frac{E_{1}^{\beta}}{\Gamma(\beta+1)}\right)^{5}
\end{aligned}
$$

from which we get that, for $n \geq 2$, the following representation holds true

$$
\frac{E_{n+1}^{\beta}}{\Gamma((n+1) \beta+1)}=\frac{(-2)^{n / 2}}{2}\left(\frac{E_{1}^{\beta}}{\Gamma(\beta+1)}\right)^{\frac{n}{2}+1}\left(\prod_{j=1}^{n / 2} \frac{\Gamma(2 j \beta+1)}{\Gamma((2 j+1) \beta+1)}+R_{n}\right) .
$$

Since

$$
A_{j}:=\frac{\Gamma(2 j \beta+1)}{\Gamma((2 j+1) \beta+1)}<A_{j-1}
$$

we obtain that

$$
R_{n} \leq 2^{n / 2} \prod_{j=1}^{n / 2} \frac{\Gamma(2 j \beta+1)}{\Gamma((2 j+1) \beta+1)}
$$

and therefore, we have that

$$
\left|\frac{E_{n+1}^{\beta}}{\Gamma((n+1) \beta+1)}\right| \leq 4^{n / 2}\left(\frac{E_{1}^{\beta}}{\Gamma(\beta+1)}\right)^{\frac{n}{2}+1} \prod_{j=1}^{n / 2} \frac{\Gamma(2 j \beta+1)}{\Gamma((2 j+1) \beta+1)} .
$$

From (3.5), we also obtain that

$$
\prod_{j=1}^{n / 2} \frac{\Gamma(2 j \beta+1)}{\Gamma((2 j+1) \beta+1)} \leq\left(\frac{\Gamma(2 \beta+1)}{\Gamma(3 \beta+1)}\right)^{n / 2}
$$

and therefore,

$$
\left|\frac{E_{n+1}^{\beta}}{\Gamma((n+1) \beta+1)}\right| \leq \frac{E_{1}^{\beta}}{\Gamma(\beta+1)}\left(4 \frac{E_{1}^{\beta}}{\Gamma(\beta+1)} \frac{\Gamma(2 \beta+1)}{\Gamma(3 \beta+1)}\right)^{n / 2} .
$$

Since $E_{2 k}^{\beta}=0$, further on we write $E_{2 k+1}^{\beta}$ instead of $E_{n+1}^{\beta}$. We now continue our analysis from (3.6) and obtain two related results:

(i) The first result immediately follows by considering that

$$
\sum_{k \geq 1}\left|\frac{E_{2 k+1}^{\beta}}{\Gamma((2 k+1) \beta+1)}\right| \frac{t^{\beta(2 k+1)}}{M^{\beta(2 k+1)}} \leq \frac{E_{1}^{\beta}}{\Gamma(\beta+1)} \frac{t^{\beta}}{M^{\beta}} \sum_{k \geq 1} a_{k} \frac{t^{2 \beta k}}{M^{2 \beta k}}
$$

where

$$
a_{k}:=\left(4 \frac{E_{1}^{\beta}}{\Gamma(\beta+1)} \frac{\Gamma(2 \beta+1)}{\Gamma(3 \beta+1)}\right)^{k}
$$

and, we recall that $E_{1}^{\beta}=1 / 4$. Then, the majorant series converges uniformly in $\left(0, r_{\beta}\right)$ and

$$
r_{\beta}=M\left(\frac{\Gamma(2 \beta+1)}{\Gamma(\beta+1) \Gamma(3 \beta+1)}\right)^{-\frac{1}{2 \beta}} \text { with } \beta \in(0,1)
$$

which increases as $\beta \uparrow 1$. Without loss of generality we consider $M=1$. Thus, $r_{\beta} \rightarrow \sqrt{3} \approx 1.732051$ as $\beta \rightarrow 1$.

(ii) By taking into account (2.12) and the left-hand side of (2.11) for $1 / \Gamma(\beta+1)$, we get that

$$
\left|\frac{E_{2 k+1}^{\beta}}{\Gamma((2 k+1) \beta+1)}\right| \leq \frac{E_{1}^{\beta}}{\Gamma(\beta+1)}\left(\frac{2}{2^{\beta}}\left(\frac{2 \beta+1}{3 \beta+1}\right)^{2 \beta+1 / 2} \frac{e^{\beta}}{(3 \beta+1)^{\beta+1 / 2-\gamma}}\right)^{k}=: b_{k} .
$$


The series

$$
\sum_{k \geq 1} b_{k} \frac{t^{\beta(2 k+1)}}{M^{\beta(2 k+1)}}
$$

uniformly converges in $\left(0, r_{\beta}\right)$ with $\beta \in(0,1)$ and

$$
r_{\beta}=M\left(\frac{2^{\beta-1}}{e^{\beta}}\left(\frac{3 \beta+1}{2 \beta+1}\right)^{2 \beta+1 / 2}(3 \beta+1)^{\beta+(1 / 2-\gamma)}\right)^{\frac{1}{2 \beta}} .
$$

For $M=1$, we have that $r_{\beta} \uparrow 1.647524$ as $\beta \rightarrow 1$.

\subsection{Integro-differential equation}

Let us focus on the Sonine kernels

$$
\kappa(t)=\frac{t^{-\beta}}{\Gamma(1-\beta)}, \quad \bar{\kappa}(t)=\frac{t^{\beta-1}}{\Gamma(\beta)}
$$

for which we have that

$$
(\kappa * \bar{\kappa})(t):=\int_{0}^{t} \kappa(s) \bar{\kappa}(t-s) d s=\frac{1}{\Gamma(1-\beta) \Gamma(\beta)} \int_{0}^{1} s^{(1-\beta)-1}(1-s)^{\beta-1} d s=1 .
$$

By considering the Laplace transform techniques, we immediately have that

$$
\left({ }^{*} D_{t}^{\alpha} u * \bar{\kappa}\right)(t)={ }^{*} D_{t}^{\alpha-\beta} u
$$

and

$$
\frac{d}{d t}\left({ }^{*} D_{t}^{\beta} u * \bar{\kappa}\right)(t)=\frac{d u}{d t} .
$$

The Eq. (3.4) can be therefore rewritten as

$$
\frac{d w}{d t}=\frac{1}{M^{\beta}} \frac{1}{\Gamma(\beta)} \int_{0}^{t}\left(w(s)-w^{2}(s)\right)(t-s)^{\beta-1} d s
$$

where a singular kernel is involved. We underline that the function (3.1) solves the integro-differential equation (3.8) and the fractional logistic equations (3.4).

Roughly speaking, we can study the solution to $\mathfrak{D}_{t}^{\Phi} u=f(u)$ by considering the equation $u^{\prime}=\bar{\kappa}^{\Phi} * f(u)$ where $\bar{\kappa}^{\Phi}$ is a kernel depending on the symbol $\Phi$.

\subsection{The Euler's $\Phi$-numbers}

The Euler's $\beta$-numbers have been introduced in the previous section as the sequence

$$
E_{0}^{\beta}=1 / 2, \quad E_{1}^{\beta}=1 / 4, \quad E_{k+1}^{\beta} \text { as in (3.2) for } k \in 2 \mathbb{N}
$$

such that

$$
w(t)=\sum_{k \geq 0} E_{k}^{\beta} \frac{t^{\beta k}}{\Gamma(\beta k+1)}
$$

solves the fractional logistic equation

$$
{ }^{*} D_{t}^{\beta} w=w-w^{2} .
$$

The Euler's $\Phi$-numbers can be therefore defined as the sequence $\left\{E_{k}^{\Phi}\right\}_{k \in \mathbb{N}_{0}}$ such that

$$
v(t)=\sum_{k \geq 0} E_{k}^{\Phi} \frac{t^{\beta k}}{\Gamma(\beta k+1)}
$$

solves the fractional logistic equation

$$
\mathfrak{D}_{t}^{\Phi} v=v-v^{2} .
$$

We provide below some examples concerning the equation with specific $\Phi$. 


\subsubsection{The Caputo-Fabrizio operator}

Recently, in a series of papers starting from [24], some authors considered the Caputo-Fabrizio operator (for a given constant $a$ )

$$
\mathscr{D}_{t}^{(\alpha)} f(t)=\frac{M(\alpha)}{1-\alpha} \int_{a}^{t} \dot{f}(\tau) \exp \left[-\frac{\alpha(t-\tau)}{1-\alpha}\right] d \tau
$$

where $M(\alpha)$ is a normalizing function such that $M(0)=M(1)=1$ and $\alpha \in(0,1)$ is the fractional order of the derivative (in the Caputo-Fabrizio notation). As pointed out in [24], we have that $\mathscr{D}_{t}^{(\alpha)} f(t) \rightarrow \dot{f}(t)$ as $\alpha \rightarrow 1$ and $\mathscr{D}_{t}^{(\alpha)} f(t) \rightarrow f(t)-f(a)$ as $\alpha \rightarrow 0$. The authors provided some properties of $\mathscr{D}_{t}^{(\alpha)} f(t)$, in particular, for $a=0$, the Laplace transform reads as follows (as usual we denote by $f$ the Laplace transform of $f$ )

$$
\begin{aligned}
\int_{0}^{\infty} e^{-\lambda t} \mathscr{D}_{t}^{(\alpha)} f(t) d t & =\frac{M(\alpha)}{1-\alpha}(\lambda \widetilde{f}(\lambda)-f(0)) \int_{0}^{\infty} e^{-\lambda t} e^{-\frac{\alpha t}{1-\alpha}} d t \\
& =\frac{M(\alpha)}{\alpha+\lambda(1-\alpha)}(\lambda \widetilde{f}(\lambda)-f(0)) .
\end{aligned}
$$

Notice that, for $\alpha=1 / 2$, the Laplace transform takes the form

$$
\int_{0}^{\infty} e^{-\lambda t} \mathscr{D}_{t}^{(1 / 2)} f(t) d t=2 \frac{M(1 / 2)}{1+\lambda}(\lambda \tilde{f}(\lambda)-f(0))
$$

that is, up to the constant $2 M(1 / 2)$, the Laplace transform of the derivative $\mathfrak{D}_{t}^{\Phi}$ with

$$
\Phi(\lambda)=\frac{\lambda}{1+\lambda} \text { and } \Pi((z, \infty))=e^{-z} .
$$

Let us consider the operator

$$
\mathfrak{D}_{t}^{\Phi} u(t)=\int_{0}^{t} u^{\prime}(s) e^{-(t-s)} d s
$$

and the fractional non-linear equation $u=\mathfrak{D}_{t}^{\Phi} U$ where $U(t)=\int_{0}^{t}\left(u(s)-u^{2}(s)\right) d s$, that is

$$
u(t)=\int_{0}^{t} u(s)(1-u(s)) e^{-(t-s)} d s .
$$

From Eq. (3.10) we get that

$$
u^{\prime}(t)=u(t)(1-u(t))-\int_{0}^{t} u(s)(1-u(s)) e^{-(t-s)} d s
$$

and

$$
u^{\prime}+u=u(1-u)
$$

which can be reduced to $u^{\prime}=-u^{2}$. Let us focus now on the solution $u$ of the logistic equation $u^{\prime}=u(1-u)$. From (3.9), we write

$$
\mathfrak{D}_{t}^{\Phi} u(t)=\int_{0}^{t} u(s)(1-u(s)) e^{-(t-s)} d s
$$

and

$$
\frac{d}{d t} \mathfrak{D}_{t}^{\Phi} u+\mathfrak{D}_{t}^{\Phi} u=u(1-u)
$$

By considering the Caputo-Fabrizio notation, we have that the solution to

$$
\mathscr{D}_{t}^{(3 / 2)} u+\mathscr{D}_{t}^{(1 / 2)} u=u(1-u)
$$

is also the solution to $u^{\prime}=u(1-u)$. In our view, find the solution to the telegraph-like logistic equation is an interesting open problem. For the linear case $\partial_{t}^{2 \beta} u+\partial_{t}^{\beta} u=\partial_{x}^{2} u$ the solution is associated with the motion of particle moving with finite velocity $[25,26]$. We provide in Fig. 1 the profile of the numerical solution to the telegraph-like equation

$$
u^{\prime \prime}+u^{\prime}=u(1-u)
$$

Let us consider the fractional integral

$$
(\mathcal{I} u)(t)=\frac{1}{\sqrt{\pi}} \int_{0}^{t}\left((t-s)^{-1 / 2}+2(t-s)^{1 / 2}\right) u(s) d s
$$




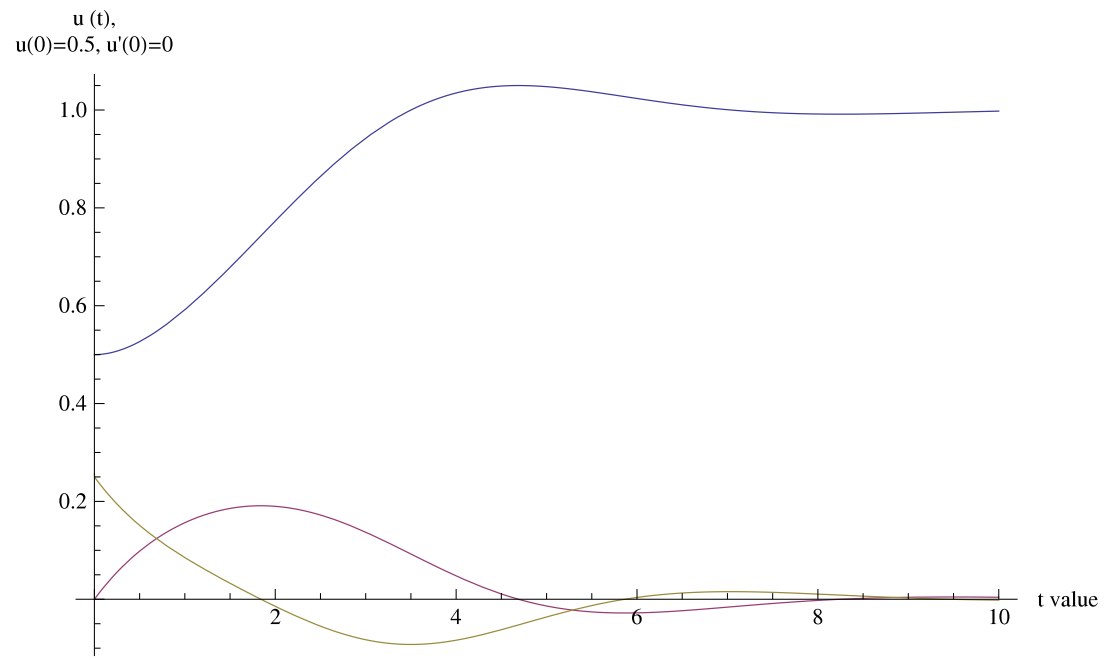

Fig. 1. The upper profile shows the numerical solution to (3.11) with $u(0)=1 / 2$ and below, $u^{\prime}$ are $u^{\prime \prime}$ are respectively plotted.

for which we have that (for a well-defined function $u$ )

$$
\int_{0}^{\infty} e^{-\lambda t}(\mathcal{I} u)(t) d t=\frac{1+\lambda}{\lambda^{3 / 2}}=\frac{1+\lambda}{\lambda} \frac{1}{\lambda^{1 / 2}} .
$$

By considering the Riemann-Liouville derivative $D_{t}^{\alpha}$ of order $\alpha=1 / 2$, we have that

$$
\int_{0}^{\infty} e^{-\lambda t}\left(D_{t}^{1 / 2} \mathcal{I} u\right)(t) d t=\frac{1+\lambda}{\lambda}=\frac{1}{\Phi(\lambda)} .
$$

This allow us to study the equation

$$
\mathfrak{D}_{t}^{\Phi} u=u(1-u)
$$

by considering the equation

$$
u^{\prime}=D_{t}^{1 / 2} \mathcal{I} u(1-u)
$$

The detailed discussion on the previous arguments will be considered in a forthcoming work.

\subsubsection{Tempered derivatives}

The tempered fractional calculus has both mathematical and practical advantages. We consider, for $\beta \in(0,1)$ and $\mu>0$,

$$
\Phi(\lambda)=\left(\mu^{\beta}-(\mu+\lambda)^{\beta}\right) \text { with } \Pi((z, \infty))=\frac{\beta}{\Gamma(1-\beta)} \int_{z}^{\infty} \frac{e^{-\mu s}}{s^{\beta+1}} d s
$$

so that $\mathfrak{D}_{t}^{\Phi}$ is the so-called tempered derivative usually denoted by $D_{t}^{\beta, \mu}$, that is by also considering the tempering parameter $\mu$ (see $[27,28]$ and the references therein for a detailed discussion). This is a special case in which in the integro-differential equation we have a kernel belonging in the well-known class of Kawashima functions. This kind of kernels are considered in the analysis of viscoelasticity with fading memory [29].

\section{Acknowledgements}

We thank Sima Sarv Ahrabi for pointing out to the authors attention the paper [8] and for the attentive reading showing some imprecisions in the previous draft. Moreover, we would like to thank the referees for comments and the constructive suggestion to add some details concerning the last section.

\section{References}

[1] P.F. Verhulst, Notice sur la loi que la population suit dans son accroissement. correspondance mathematique et physique publié par a, Quetelet (Brussels) X (1838) 113-121.

[2] B.-Q. Ai, X.-J. Wang, G.-T. Liu, L.-G. Liu, Correlated noise in a logistic growth model, Phys. Rev. E (3) 67 (2003) 229031-229033.

[3] A. Belmiloudi, Mathematical modelling and optimal control problems in brain tumour targeted drug delivery strategies, Internat. J. Biomath. 10 (2017). 
[4] R.A. Ku-Carrillo, S.E. Delgadillo, M.B. Chen-Charpentier, A mathematical model for the effect of obesity on cancer growth and on the immune system response, Appl. Math. Model. 40 (2016) 4908-4920.

[5] D. Kirschner, J. Panetta, Modeling immunotherapy of the tumor-immune interaction, J. Math. Biol. 37 (1998) 235-252.

[6] H.R. Thieme, Mathematics in Population Biology, in: Princeton Series in Theoretical and Computational Biology, Princeton University Press, 2003.

[7] M. D'Ovidio, P. Loreti, A. Momenzadeh, S.S. Ahrabi, Determination of order in linear fractional differential equations (submitted for publication). arXiv: 171001132.

[8] I. Area, J. Losada, J.J. Nieto, A note on the fractional logistic equation, Physica A 444 (2016) 182-187.

[9] A.M.A. El-Sayed, A.E.M. El-Mesiry, H.A.A. El-Saka, On the fractional-order logistic equation, Appl. Math. Lett. 20 (2007) $817-823$.

[10] X.-J. Yang, J.A. Tenreiro Machado, A new insight into complexity from the local fractional calculus view point: modelling growths of populations, Math. Methods Appl. Sci. 40 (2017) 6070-6075.

[11] B.J. West, Exact solution to fractional logistic equation, Physica A 429 (2015) 103-108.

[12] M. Ortigueira, G. Bengochea, A new look at the fractionalization of the logistic equation, Physica A 467 (2017) 554-561.

[13] M. D'Ovidio, P. Loreti, S.S. Ahrabi, Modified fractional logistic equation, Physica A 505 (2018) 818-824.

[14] I.S. Gradshteyn, I.M. Ryzhik, Table of Integrals, Series, and Products, seventh ed., Academic Press, Elsevier inc, 2007.

[15] K. Dilcher, Asymptotic behaviour of bernoulli, euler and generalized bernoulli polynomials, J. Approx. Theory 49 (1987) 321-330.

[16] Z.-Q. Chen, Time fractional equations and probabilistic representation, Chaos Solitons Fractals 102 (2017) 168-174.

[17] R. Capitanelli, M. D'Ovidio, Fractional equations via convergence of forms, 2017 (submitted for publication). arXiv:1710.01147.

[18] B. Toaldo, Convolution-type derivatives hitting-times of subordinators and time-changed $C_{\infty}$-semigroups, Potential Anal. 42 (2015) $115-140$.

[19] A.A. Kilbas, H.M. Srivastava, J.J. Trujillo, Theory and Applications of Fractional Differential Equations, in: North-Holland Mathematics Studies, vol. 204, Elsevier, Amsterdam, 2006

[20] I. Podlubny, An Introduction to Fractional Derivatives, Fractional Differential Equations, Some Methods of their Solution and Some of their Applications, Academic Press, New York, 1999.

[21] H. Alzer, Sharp inequalities for the beta function, Indag. Math. N.S. 12 (1) (2001) 15-21.

[22] A. Laforgia, P. Natalini, On some inequalities for the gamma function, Adv. Dyn. Syst. Appl. 8 (2013) 261-267.

[23] X. Li, C.-P. Chen, Inequalities for the gamma function, J. Inequal. Pure Appl. Math. 8 (2007) 3p.

[24] M. Caputo, M. Fabrizio, A new definition of fractional derivative without singular kernel, progr, Fract. Differ. Appl. 1 (2) (2015) $73-85$.

[25] M. D'Ovidio, F. Polito, Fractional diffusion-telegraph equations and their associated stochastic solutions, Theory Probab. Appl. 62 (2017) $692-718$.

[26] M. D'Ovidio, B. Toaldo, E. Orsingher, Time changed processes governed by space-time fractional telegraph equations, Stoch. Anal. Appl. 32 (6) (2014) 1009-1045.

[27] L. Beghin, On fractional tempered stable processes and their governing differential equations, J. Comput. Phys. 293 (2015) 29-39.

[28] F. Sabzikar, M.M. Meerschaert, J. Chen, Tempered fractional calculus, J. Comput. Phys. 293 (2015) 14-28.

[29] S. Kawashima, Global solutions to the equation of viscoelasticity with fading memory, J. Differential Equations 101 (2) (1993) 388-420. 